\title{
Some Remarks on the Construction of Extended Gaussian Quadrature Rules
}

\author{
By Giovanni Monegato*
}

\begin{abstract}
We recall some results from a paper by Szegö on a class of polynomials which are related to extended Gaussian quadrature rules. We show that a very efficient algorithm, for the computation of the abscissas of the rules in question, was already described in that paper. We also point out that this method extends to rules for integrals with an ultraspherical-type weight function. A bound for the error of some of the above rules is also given.
\end{abstract}

1. Introduction. A remarkable class of integration rules has been considered by Kronrod [2] and has received some attention by others; for instance, see [3] - [6] .

Consider the $n$-point Gaussian quadrature formula

$$
\int_{-1}^{1} f(x) d x=\sum_{i=1}^{n} H_{i}^{(n)} f\left(\xi_{i}^{(n)}\right)+R_{G}^{(n)}(f)
$$

which has, as it is well known, degree of exactness $2 n-1$, i.e., $R_{G}^{(n)}(f)=0$ whenever $f$ is a polynomial of degree at most $2 n-1$. It is also well known that the nodes $\xi_{i}^{(n)}$ are the zeros of the Legendre polynomial of degree $n$.

In order to give an estimate of the error $R_{G}^{(n)}(f)$, Kronrod proposes to associate with (1), for comparison, a new rule which uses $2 n+1$ abscissas in $(-1,1), n$ of which are those present in (1), and has maximum degree of exactness. More precisely, he studies a quadrature formula of the type

$$
\int_{-1}^{1} f(x) d x=\sum_{i=1}^{n} A_{i}^{(n)} f\left(\xi_{i}^{(n)}\right)+\sum_{j=1}^{n+1} B_{j}^{(n)} f\left(x_{j}^{(n)}\right)+R_{K}^{(n)}(f),
$$

where $\xi_{i}^{(n)}, i=1, \ldots, n$, are the zeros of the $n$th degree Legendre polynomial $P_{n}(x)$, while the nodes $x_{j}^{(n)}$ and the weights $A_{i}^{(n)}, B_{j}^{(n)}$ are chosen so that (2) has degree of exactness $3 n+1\left(3 n+2\right.$ if $n$ is odd). If we denote by $E_{n+1}(x)$ the polynomial of degree $n+1$, whose zeros are the abscissas $x_{j}^{(n)}, j=1,2, \ldots, n+1$, then $E_{n+1}(x)$ has to satisfy the following orthogonality relation

$$
\int_{-1}^{1} P_{n}(x) E_{n+1}(x) x^{k} d x=0, \quad k=0,1, \ldots, n .
$$

The coefficients of $E_{n+1}(x)$ may be uniquely determined, up to a multiplicative constant, by solving a nonsingular triangular linear system which is derived from the defining relation (3) and the orthogonal property of $P_{n}(x)$. Szegö [7] has also shown that the zeros of $E_{n+1}(x)$ are in $(-1,1)$ and alternate with those of $P_{n}(x)$.

Received April 21, 1977.

AMS (MOS) subject classifications (1970). Primary 65D30; Secondary 33A65.

*Work performed under the auspices of the Italian Research Council. 
Kronrod computes the coefficients of $E_{n+1}(x)$ by solving the linear triangular system we have just mentioned, which is very ill-conditioned. A much better algorithm is given by Patterson [4], who expands $E_{n+1}(x)$ in terms of Legendre polynomials and obtains a well-conditioned linear system of equations. More recently, Piessens and Branders [6] propose to expand $E_{n+1}(x)$ in a series of Chebyshev polynomials, and give an algorithm which is the most efficient. All three methods were directly derived from (3).

In the next section, we first outline some results from the paper by Szegö [7]. We then remark that the method presented by Piessens and Branders can be derived in a different way, as it had already been done in [7]. As a matter of fact, Szegö gives a method which is valid for a wider class of polynomials $E_{n+1}(x)$, arising when in (1) and (2) a weight function of the type $\left(1-x^{2}\right)^{\mu-1 / 2}$ is present. This same method can, for example, be used to derive the extension of Lobatto rules. An estimate for $\left|R_{K}^{(n)}(f)\right|$ is also given when $0<\mu \leqslant 1$.

2. Szegö's Results. Let us consider Legendre's differential equation

$$
\left(1-x^{2}\right) y^{\prime \prime}-2 x y^{\prime}+n(n+1) y=0 .
$$

It is well known that the $n$th degree Legendre polynomial $P_{n}(x)$ is, up to a constant factor, the unique polynomial solution of (4). Let $x$ be arbitrary in the complex plane cut along the segment $[-1,1]$ and $n \geqslant 1$; then a second solution of (4), which is linearly independent of $P_{n}(x)$, is the so-called Legendre function of the second kind

$$
Q_{n}(x)=\frac{1}{2} \int_{-1}^{1} \frac{P_{n}(t)}{x-t} d t=\frac{c_{1}}{x^{n+1}}+\frac{c_{2}}{x^{n+3}}+\ldots
$$

with the last expansion convergent for $|x|>1$. We thus have

$$
\left[Q_{n}(x)\right]^{-1}=E_{n+1}(x)+\frac{a_{1}}{x}+\frac{a_{2}}{x^{2}}+\ldots, \quad n \geqslant 1,
$$

where $E_{n+1}(x)$ is a polynomial of degree $n+1$. It can be shown [7] that $E_{n+1}(x)$ satisfies the orthogonality relation (3); hence, it is the polynomial we are looking for. Moreover, from (5), (6) and the fact that $Q_{n}(x)$ satisfies a three-term recurrence relation, it also follows [7] that

$$
Q_{n}(x) E_{n+1}(x)=1+b_{0} Q_{n+1}(x)+b_{1} Q_{n+2}(x)+\cdots+b_{n} Q_{2 n+1}(x) .
$$

Let us introduce the function

$$
\bar{Q}_{n}(x)=1 / 2\left[Q_{n}(x+i 0)+Q_{n}(x-i 0)\right],
$$

which is analytic on $(-1,1)$ and satisfies (4). The following convergent expansion is known [8, p. 92]

$$
\bar{Q}_{n}(\cos \phi)=2 \frac{2 \cdot 4 \cdots 2 n}{3 \cdot 5 \cdot \cdot(2 n+1)} \sum_{\nu=0}^{\infty} f_{\nu} \cos (n+2 \nu+1) \phi, \quad 0<\phi<\pi
$$


where

$$
f_{0}=1, \quad f_{\nu}=\frac{1 \cdot 3 \cdots(2 \nu-1)}{2 \cdot 4 \cdots 2 \nu} \frac{(n+1)(n+2) \cdots(n+\nu)}{(n+3 / 2)(n+5 / 2) \cdots(n+\nu+1 / 2)},
$$

In his paper, Szegö sets

$$
v=1,2, \ldots
$$

(10) $E_{n+1}(\cos \phi)=\lambda_{0} \cos (n+1) \phi+\lambda_{1} \cos (n-1) \phi+\cdots+ \begin{cases}\lambda_{n / 2} \cos \phi, & n \text { even, } \\ 1 / 2 \lambda_{(n+1) / 2}, & n \text { odd; }\end{cases}$ then, by means of (7), (8) and (9), he deduces that the coefficients $\lambda_{i}$ are the solution of the following triangular system

$$
\left\{\begin{array}{l}
\sum_{i=0}^{k} f_{i} \lambda_{k-i}=0, \quad k=1, \ldots, m, \quad m=\left[\frac{n+1}{2}\right], \\
\frac{2 \cdot 4 \cdot 2 n}{3 \cdot 5 \cdots(2 n+1)} f_{0} \lambda_{0}=1 .
\end{array}\right.
$$

Let us rewrite (10) in the following form

$$
\bar{E}_{n+1}(x)=\frac{1}{\lambda_{0}} E_{n+1}(x)
$$

$$
=T_{n+1}(x)+\alpha_{1} T_{n-1}(x)+\cdots+ \begin{cases}\alpha_{n / 2} T_{1}(x), & n \text { even, } \\ 1 / 2 \alpha_{(n+1) / 2}, & n \text { odd, }\end{cases}
$$

where $x=\cos \phi$ and $T_{k}(x)$ is the $k$ th degree Chebyshev polynomial; then, from (11) and $\left(9^{\prime}\right)$, we have

$$
\left\{\begin{array}{l}
\alpha_{1}=-f_{1} \\
\alpha_{k}=-f_{k}-\sum_{i=1}^{k-1} f_{i} \alpha_{k-i}, \quad k=2, \ldots, m
\end{array}\right.
$$

and

$$
\left\{\begin{array}{l}
f_{1}=(n+1) /(2 n+3) \\
f_{k+1}=\frac{(2 k+1)(n+k+1)}{(k+1)(2 n+2 k+3)} f_{k}, \quad k=1, \ldots, m-1 .
\end{array}\right.
$$

We now observe that (13) is identical with system (6) of Piessens and Branders [6], and the form of the recursive relation (14) is simpler than the corresponding one presented in [6].

In his paper, Szegö has extended his results to certain ultraspherical polynomials. If we denote by $P_{n}^{(\mu)}(x), \mu>-1 / 2$, the ultraspherical polynomial of degree $n$, orthogonal on $[-1,1]$ with respect to the weight function $\left(1-x^{2}\right)^{\mu-1 / 2}$, and with $Q_{n}^{(\mu)}(x)$ the corresponding function of the second kind 


$$
\left(1-x^{2}\right)^{\mu-1 / 2} Q_{n}^{(\mu)}(x)=\frac{1}{2} \frac{\Gamma(2 \mu)}{\Gamma(\mu+1 / 2)} \int_{-1}^{1}\left(1-t^{2}\right)^{\mu-1 / 2} \frac{P_{n}^{(\mu)}(t)}{x-t} d t, \quad n \geqslant 1,
$$

then, by an argument analogous to the Legendre case, we have

$$
\left[\left(1-x^{2}\right)^{\mu-1 / 2} Q_{n}^{(\mu)}(x)\right]^{-1}=E_{n+1}^{(\mu)}(x)+\frac{a_{1}^{(\mu)}}{x}+\frac{a_{2}^{(\mu)}}{x^{2}}+\ldots,
$$

where $E_{n+1}^{(\mu)}(x)$ satisfies the orthogonality relation

$$
\int_{-1}^{1}\left(1-x^{2}\right)^{\mu-1 / 2} P_{n}^{(\mu)}(x) E_{n+1}^{(\mu)}(x) x^{k} d x=0, \quad k=0,1, \ldots, n .
$$

In a way much similar to the Legendre case, Szegö derives the following expansion,

$$
\left(1-\cos ^{2} \phi\right)^{\mu-1 / 2} \bar{Q}_{n}^{(\mu)}(\cos \phi)=\sqrt{\pi} \frac{\Gamma(n+2 \mu)}{\Gamma(n+\mu+1)} \sum_{\nu=0}^{\infty} f_{\nu}^{(\mu)} \cos (n+2 \nu+1) \phi,
$$

$$
f_{0}^{(\mu)}=1, \quad f_{\nu+1}^{(\mu)}=\frac{(\nu+1-\mu)(n+\nu+1)}{(\nu+1)(n+\nu+1+\mu)} f_{\nu}^{(\mu)}, \quad \nu=0,1, \ldots,
$$

where $\bar{Q}_{n}^{(\mu)}(\cos \phi)$ is the ultraspherical analogue of $\bar{Q}_{n}(\cos \phi)$.

Let

$E_{n+1}^{(\mu)}(\cos \phi)=\lambda_{0}^{(\mu)} \cos (n+1) \phi+\lambda_{1}^{(\mu)} \cos (n-1) \phi+\cdots+ \begin{cases}\lambda_{n / 2}^{(\mu)} \cos \phi, & n \text { even, } \\ 1 / 2 \lambda_{(n+1) / 2}^{(\mu)}, & n \text { odd }\end{cases}$

Szegö [7] has proved that for $0<\mu \leqslant 2$, the zeros of $E_{n+1}^{(\mu)}(x)$ are all distinct and in $(-1,1)$; moreover, they interlace with the zeros of $P_{n}^{(\mu)}(x)$. He has also proved the following properties of the coefficients $\lambda_{i}^{(\mu)}$ :

(i) $0<\mu<1, \lambda_{0}^{(\mu)}>0, \lambda_{i}^{(\mu)}<0, i=1, \ldots, m$,

$$
\lambda_{0}^{(\mu)}>-\sum_{i=1}^{m} \lambda_{i}^{(\mu)}
$$

(ii) $1<\mu<2, \lambda_{i}^{(\mu)}>0, i=0,1, \ldots, m$;

(iii)

$$
\begin{array}{ll}
\mu=0, & E_{2}^{(0)}(\cos \phi)=\cos 2 \phi-1 / 2, \\
& E_{n+1}^{(0)}(\cos \phi)=\frac{2 n}{\sqrt{\pi}}[\cos (n+1) \phi-\cos (n-1) \phi], \quad n \geqslant 2 ; \\
\mu=1, \quad E_{n+1}^{(1)}(\cos \phi)=\frac{2}{\sqrt{\pi}} \cos (n+1) \phi ; \\
\mu=2, \quad \lambda_{i}^{(2)}=\frac{2}{\sqrt{\pi}} \frac{1}{n+3}\left(\frac{n+1}{n+3}\right)^{i}, \quad i=0,1, \ldots, m .
\end{array}
$$

As before in the Legendre case, we write 


$$
\begin{aligned}
\bar{E}_{n+1}^{(\mu)}(x) & =\frac{1}{\lambda_{0}^{(\mu)}} E_{n+1}^{(\mu)}(x) \\
& =T_{n+1}(x)+\alpha_{1}^{(\mu)} T_{n-1}(x)+\cdots+\left\{\begin{aligned}
\alpha_{n / 2}^{(\mu)} T_{1}(x), & n \text { even, } \\
1 / 2 \alpha_{(n+1) / 2}^{(\mu)}, & n \text { odd, } \\
\mu>-1 / 2, & n \geqslant 1, \quad x=\cos \phi .
\end{aligned}\right.
\end{aligned}
$$

The coefficients $\alpha_{i}^{(\mu)}$ are again the solution of system (13) with $f_{\nu}^{(\mu)}, \nu=0,1, \ldots$, $m$, given by means of (15).

A particular case is the extension of Lobatto quadrature rules, considered by Patterson [5] and Piessens and Branders [6], which is easily obtained by letting $\mu=$ $3 / 2$. The algorithm is again essentially identical with the one presented in [6], but the recursive relation (15) is simpler than the corresponding one given in that paper.

Similarly, we may construct extensions of Lobatto-type quadrature rules with multiple nodes at the endpoints of the interval of integration, when they exist.

3. An Error Estimate. From the properties of the coefficients $\lambda_{i}^{(\mu)}$ we may derive that, when $0 \leqslant \mu \leqslant 1$,

$$
\left|\widetilde{E}_{n+1}^{(\mu)}(x)\right|<2, \quad-1 \leqslant x \leqslant 1 .
$$

This is sufficient to give an estimate of $\left|R_{K}^{(n)}(f)\right|$ when $f$ is a sufficiently smooth function.

Let us suppose $f \in C^{(3 n+2)}[-1,1]$; then

$$
R_{K}^{(n)}(f)=\frac{1}{2^{2 n} K_{n}^{(\mu)}(3 n+2) !} \int_{-1}^{1}\left(1-x^{2}\right)^{\mu-1 / 2} P_{n}^{(\mu)}(x)\left[\bar{E}_{n+1}^{(\mu)}(x)\right]^{2} f^{(3 n+2)}\left(\xi_{x}\right) d x
$$

where

$$
K_{n}^{(\mu)}=\frac{2^{n}}{n !} \frac{\Gamma(n+\mu)}{\Gamma(\mu)}
$$

We also know that, for $\mu>0$,

$$
\max _{-1<x<1}\left|P_{n}^{(\mu)}(x)\right|=\left(\begin{array}{c}
n+2 \mu-1 \\
n
\end{array}\right)
$$

so that from (16), when $0<\mu \leqslant 1$ we get

$$
\left|R_{K}^{(n)}(f)\right|<\frac{\pi \Gamma(n+2 \mu)}{2^{3 n+2 \mu-3}(3 n+2) ! \Gamma(\mu+1) \Gamma(n+\mu)} M_{3 n+2},
$$

with $M_{3 n+2}=\max _{-1<x<1}\left|f^{(3 n+2)}(x)\right|$.

In much the same way, when $n$ is odd, if we assume $f \in C^{(3 n+3)}[-1,1]$, we may derive the same bound for $\left|R_{K}^{(n)}(f)\right|$, where $M_{3 n+2}$ has to be replaced by $M_{3 n+3}$ $=\max _{-1<x<1}\left|f^{(3 n+3)}(x)\right|$ and $(3 n+2) !$ by $(3 n+3) !$. 
1. P. J. DAVIS \& P. RABINOWITZ, Methods of Numerical Integration, Academic Press, New York, 1975.

2. A. S. KRONROD, Nodes and Weights for Quadrature Formulae. Sixteen-Place Tables, "Nauka", Moscow, 1964; English transl., Consultants Bureau, New York, 1965. MR 32 \#597, \#598.

3. G. MONEGATO, "A note on extended Gaussian quadrature rules," Math. Comp., v. 30, 1976, pp. $812-817$.

4. T. N. L. PATTERSON, "The optimum addition of points to quadrature formulae," Math. Comp., v. 22, 1968, pp. 847-856; Addendum, ibid., v. 22, 1968, no. 104, loose microfiche suppl. C1-C11. MR 39 \#3701.

5. T. N. L. PATTERSON, "On some Gauss and Lobatto based quadrature formulae,"

Math. Comp., v. 22, 1968, pp. 877-881; Addendum, ibid., v. 22, 1968, no. 104, loose microfiche suppl. D1-D5.

6. R. PIESSENS \& M. BRANDERS, "A note on the optimal addition of abscissas to quadrature formulas of Gauss and Lobatto ty pe," Math. Comp., v. 28, 1974, pp. 135-139.

7. G. SZEGÖ, "Über gewisse orthogonale Polynome, die zu einer oszillierenden Belegungsfunktion gehören," Math. Ann., v. 110, 1934, pp. 501-513.

8. G. SZEGÖ, Orthogonal Polynomials, Amer. Math. Soc. Colloq. Publ., vol. 23, 4th ed., Amer. Math. Soc., Providence, R. I., 1975. 